\title{
FÍSTULAS VESICOUTERINAS. NUESTRA EXPERIENCIA TRAS 25 AÑOS
}

\author{
M.A. BONILLO GARCÍA, J.J. PACHECO BRU, J.L. PALMERO MARTÍ, \\ J.M. ALAPONT ALACREU, M. ALONSO GORREA, S. ARLANDIS GUZMÁN, \\ J.F. JIMÉNEZ CRUZ
}

\author{
Servicio de Urología. Hospital Universitario La Fe. Valencia. \\ Actas Urol Esp. 27 (9): 707-712, 2003
}

\section{RESUMEN}

FÍSTULAS VESICOUTERINAS. NUESTRA EXPERIENCIA TRAS 25 AÑOS

OBJETIVO: Determinar el estado actual de las fistulas vesicouterinas en nuestro hospital, los factores asociados a su desarrollo y evaluar los resultados de los tratamientos realizados para su resolución.

MATERIAL Y MÉTODOS: Realizamos un estudio retrospectivo de las pacientes con fístulas vesicouterinas tratadas en nuestro servicio en los últimos 25 años con un seguimiento mínimo de 2 años.

RESULTADOS: Se evaluaron 6 casos de fístulas vesicouterinas. En el 83,3\% (5/6) de los casos la causa principal fue la realización de una cesárea. La edad media de las mujeres fue de 36,5 años. En el 50\% de los casos la incontinencia urinaria inmediata a la intervención quirúrgica condicionó el diagnóstico precoz. En 5 pacientes se realizó una reparación quirúrgica diferida de la fístula vía abdominal. Todas ellas permanecieron asintomáticas durante los años de seguimiento. En 2 pacientes se produjo un embarazo a término a partir de los 24 meses de la intervención.

CONCLUSIONES: La fístula vesicouterina, aún siendo infrecuente, ha dejado de ser un diagnóstico excepcional. Las cesáreas segmentarias bajas constituyen actualmente el mayor factor de riesgo aislado relacionado con el desarrollo de estas fístulas. Recomendamos una reparación quirúrgica diferida sin descartar una actitud conservadora en los casos de pequeño tamaño diagnosticados precozmente.

PALABRAS CLAVE: Fístulas vesicouterinas. Cesárea. Incontinencia urinaria.

\section{ABSTRACT}

VESICOUTERINE FISTULAE. 25 YEARS OF OUR EXPERIENCE

OBJECTIVE: To determine the current state of vesicouterine fistulae in our hospital, the factors associated with their development and the findings obtained from the treatments used.

MATERIAL AND METHODS: We carry out a retrospective study on a group of patients suffering from vesicouterine fistulae who were treated in our service for the last 25 years with a 2 year minimum follow-up.

RESULTS: 6 cases of vesicouterine fistulae were evaluated. In 83,3\% (5/6) of the cases occurred following caesarean section. The average age of the patients was 36,5 years old. In $50 \%$ of the cases, urinary incontinence immediately after surgery determined an early diagnosis. In 5 patients deferred abdominal surgical approach was carried out. All of the patients remained asymptomatic during the follow-up years. Two pregnancies were recorded 24 months after repair with a full term delivery.

CONCLUSIONS: The vesicouterine fistula, despite being infrequent, is no longer an exceptional diagnosis. Currently, the low segmentarian caesareans constitute the major isolated risk factor for fistula development. We recommend a deferred surgical repair without discarding a conservative approach for those cases of small and early fistula.

KEY WORDS: Vesicouterine fistulae. Caesarean section. Urinary incontinence. 
$\mathrm{L}^{2}$ as fístulas del tracto urogenital femenino son complicaciones infrecuentes de maniobras obstétricas, patologías pélvicas o del tratamiento de las mismas. De todas ellas, la fístula vesicouterina es una de las menos comunes descrita como una comunicación anormal entre la vejiga y la cavidad uterina o el canal cervical.

La incidencia de esta fístula ha aumentado significativamente en las últimas décadas ${ }^{1}$ debido al incremento de cesáreas segmentarias bajas; desde que en 1908 Knipe $^{2}$ describiese el primer caso hasta la revisión llevada a cabo por Tancer ${ }^{3}$ en 1986 se documentaron 92 casos, mientras que en 1994 Issa et al. ${ }^{4}$ describieron 145 casos, y en 1997 Yip et al. ${ }^{2}$ notificaron un total de 156 casos. Sin considerar la exactitud de estas cifras nos encontramos ante un incremento de publicaciones en la literatura médica referentes a las fístulas vesicouterinas las cuales, al afectar principalmente a mujeres jóvenes, justifican aún más el impacto social de esta patología.

En 1957 Youssef $^{5}$ describió un síndrome clásico asociado a la aparición de esta fístula tras la realización de una cesárea segmentaria; éste consiste en la presencia de amenorrea, continencia urinaria y orina hemática de forma cíclica coincidiendo con la supuesta menstruación. Este último signo lo acuñó como menuria, es decir, eliminación de sangre menstrual a través del tracto urinario que no debe ser confundido con el término de hematuria.

Hemos llevado a cabo un estudio descriptivo para determinar el estado actual de este tipo de fístula en nuestro hospital y las situaciones asociadas a su desarrollo.

\section{MATERIAL Y MÉTODOS}

Realizamos un estudio retrospectivo de las pacientes que fueron valoradas por el Servicio de Urología del Hospital Universitario La Fe en los últimos 25 años, por presentar una fístula en el tracto urogenital bien documentada. Se identificaron un total de 6 casos de fístulas vesicouterinas. La edad media de las mujeres en el momento de la presentación de la sintomatología era de 36,5 años. Se estableció el diagnóstico tras la confirmación de la sospecha clínica tanto con la exploración física (prueba del azul de metileno), como con las técnicas de imagen (histerosalpingografía, urografía y/o cistoscopia).

\section{RESULTADOS}

En estos últimos 25 años se han constatado 6 casos de fístulas vesicouterinas $(3,60 \%)$ en el contexto de 167 fístulas del tracto urogenital femenino diagnosticadas (Tabla I) ${ }^{6,7}$. El 33,3\% (2/6) afectaban al cuerpo del útero, llamadas vesicocorporales, mientras que el 66,6\% restante (4/6) comprometían al cérvix uterino, denominándose vesicocervicales. En 2 de estos últimos casos, la fístula también comprometía la cavidad vaginal.

\section{TABLA I}

TIPOS DE FÍSTULAS GENITOURINARIAS REGISTRADAS EN LOS ÚLTIMOS 25 AÑOS

\begin{tabular}{|l|c|c|}
\hline Tipo de fístula & № & $\%$ \\
\hline Ureterovaginal & 84 & $50,29 \%$ \\
\hline Vesicovaginal & 75 & $44,91 \%$ \\
\hline Vesicouterina & 6 & $3,60 \%$ \\
\hline Uretrovaginal & 2 & $1,20 \%$ \\
\hline Total & 167 & $100 \%$ \\
\hline
\end{tabular}

En el 83,3\% (5/6) de los casos la causa principal fue la realización de una cesárea segmentaria baja; 4/6 pacientes presentaban el antecedente de al menos una cesárea previa, sólo una se sometía por primera vez a una cirugía obstétrica. Un caso fue secundario a la diseminación local de un adenocarcinoma de cérvix IIIb-IVa, asociándose a un trayecto fistuloso hacia la vagina.

En el 50\% de las pacientes, la incontinencia urinaria inmediata a la intervención quirúrgica fue lo que condicionó el diagnóstico precoz, siendo el segundo día del postoperatorio el más común en su aparición. En una de las pacientes se detectó una comunicación aberrante (vesicocorporal) en el contexto de una esterilidad secundaria, por lo demás totalmente asintomática, sin incontinencia ni menuria; en otra de las pacientes se diagnosticó durante el estudio de extensión de su proceso neoplásico mediante una RMN (Fig. 1), y se manifestó con genitorragia e intensos dolores pélvicos. Tan sólo una debutó con amenorrea, menuria sin incontinencia urinaria, que correspondería con la triada clásica descrita por Youssef (Tabla II). 


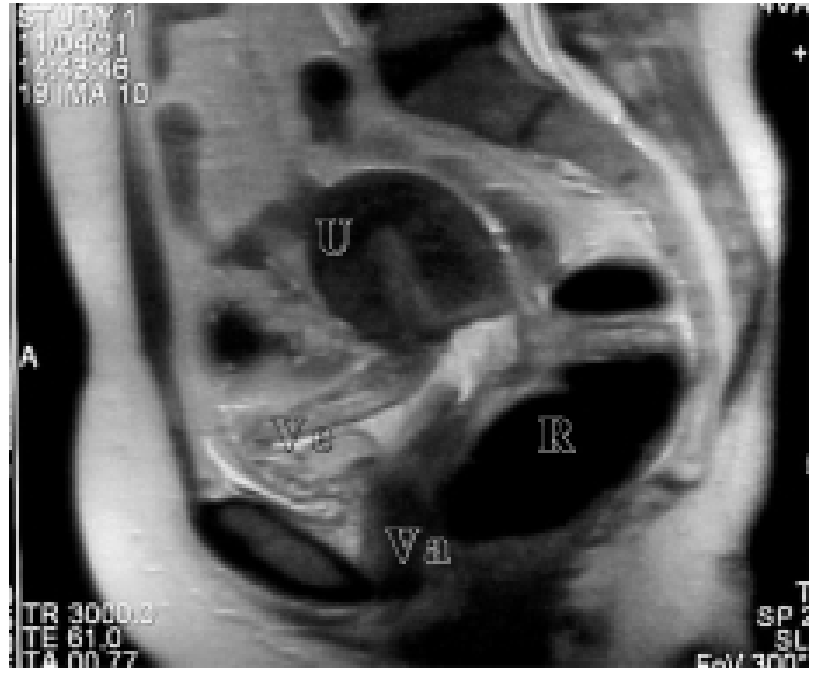

FIGURA 1. Resonancia Magnética Nuclear (RMN). Detalle de una fistula vesicocervical. Nivel hidroaéreo en el interior del útero como consecuencia de la comunicación abe rrante entre la cara anterior del cérvix uterino y la vejiga. (Ve: Vejiga; R: Recto; U: Útero; Va: Vagina).

Una vez remitida la paciente con la sospecha clínica de fístula vesicouterina, el primer paso diagnóstico es la instilación vesical de azul de metileno que aparece por vagina. Posteriormente, una histerosalpingografia (Fig. 2) muestra el paso de contraste entre las dos cavidades, vesical y uterina. El estudio urográfico (Figs. 3 y 4) descarta lesión ureteral y posibles modificaciones en la cavidad vesical. Del mismo modo, la uretrocistoscopia nos confirma un defecto de la cara posterior de la vejiga, generalmente por encima del trígono, y su relación con los meatos ureterales. Actualmente la RMN nos ofrece, con una sola exploración, el trayecto fistuloso y la situación vesico-ureteral.
En las 3 pacientes diagnosticadas precozmente tras la cirugía obstétrica se les cateterizó mediante una sonda vesical durante un periodo entre 1-2 semanas; sin embargo, al persistir la incontinencia se intervinieron quirúrgicamente durante el siguiente mes. El tratamiento conservador mediante sondaje vesical y anticonceptivos orales durante 6 meses no fue eficaz en la paciente que debutó con menuria. En la paciente remitida por estudio de esterilidad, se intentó en otro centro sin éxito el sellado de la fístula vesicouterina con Histoacryl ${ }^{\circledR}$. Las pacientes que fueron subsidiarias de un tratamiento quirúrgico $(5 / 6)$ se les realizó una reparación de la fístula vía transperitoneal, suturando el orificio vesical en dos planos y el uterino en uno, e interponiendo

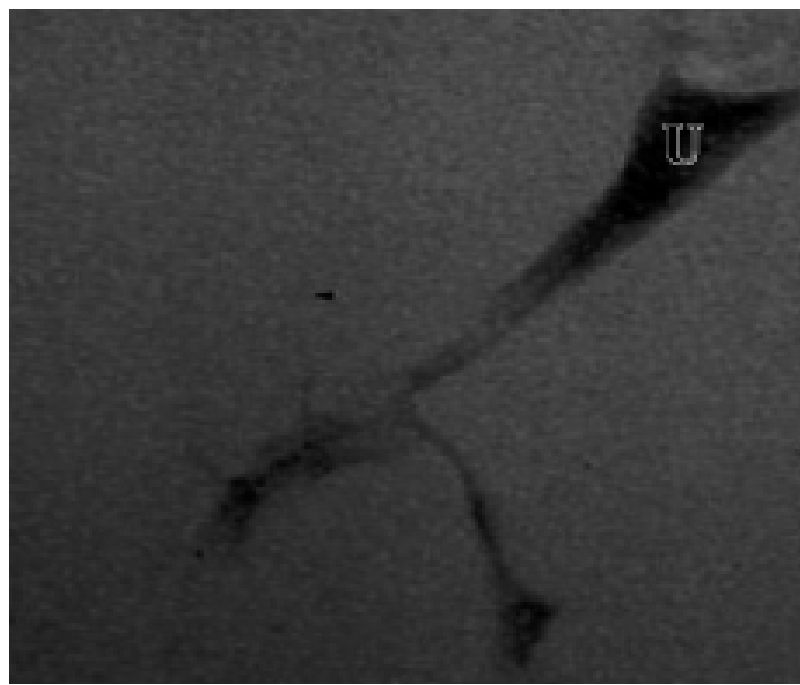

FIGURA 2. Histerosalpingografia (HSG). Paso de contraste hacia la vejiga tras instilación del mismo en la cavidad uterina. (U: Útero).

\section{TABLA II}

PACIENTES CON FÍSTULAS VESICOUTERINAS TRATADAS EN NUESTRO SERVICIO EN LOS ÚLTIMOS 25 AÑOS

\begin{tabular}{||c|c|c|c|c|c|c|}
\hline & Edad & Etiología & Clínica & Diagnóstico & Localización & Tratamiento \\
\hline 1 & 32 & Cesárea & Incontinencia & HSG (+) & Vesicocervical & CIR \\
\hline 2 & 36 & Cesárea & Esterilidad & HSG (+) & Vesicocorporal & CIR \\
\hline 3 & 48 & ADC Cérvix & Genitorragia & RMN (+) & Vesicocervical & Paliativo \\
\hline 5 & 28 & Cesárea & Menuria & HSG (+) & Vesicocorporal & CIR \\
\hline 6 & 32 & Cesárea & Incontinencia & HSG (+) & Vesicocervical & CIR \\
\hline
\end{tabular}

HSG: Histerosalpingografía 


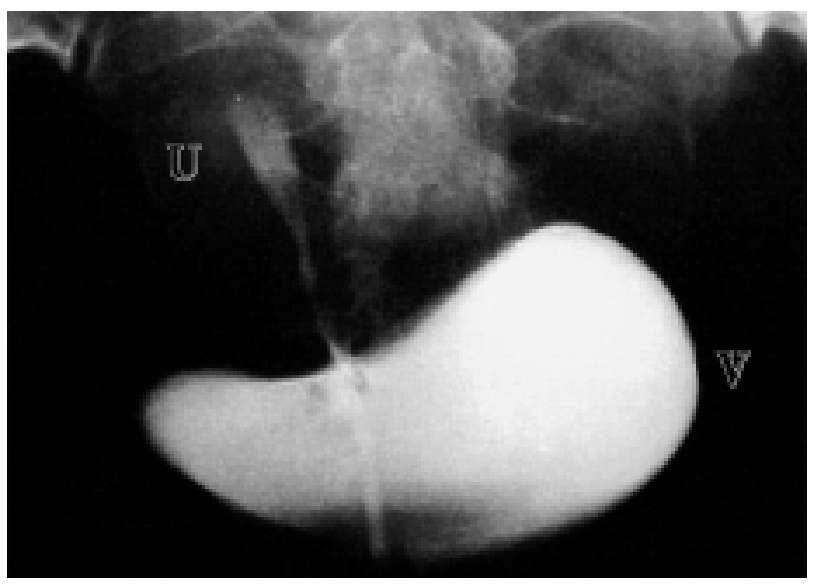

FIGURA 3. Cistografia Ascendente. Muestra la impronta en la parte superior de la vejiga y el paso de contraste hacia la cavidad uterina.

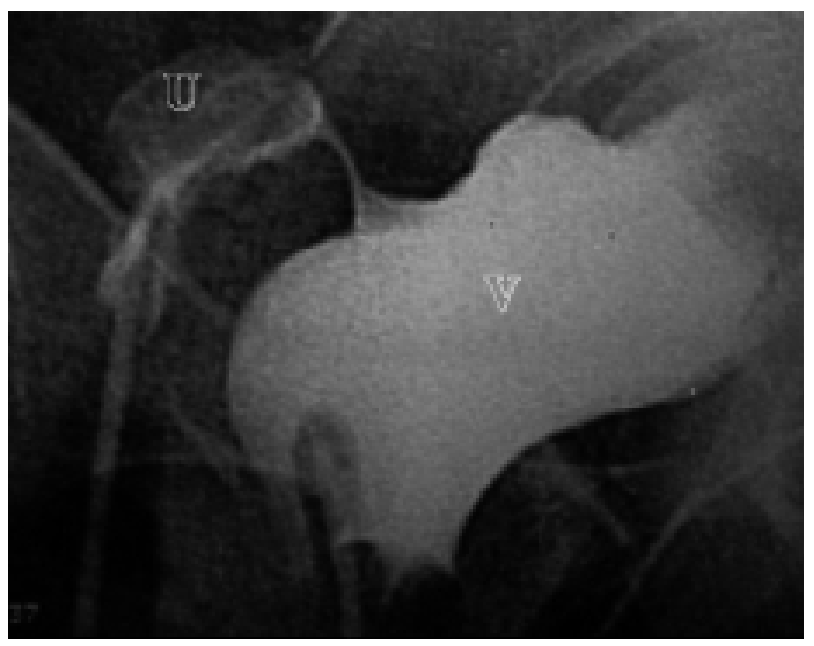

FIGURA 4. Técnica de imagen combinada Cisto + Histerosalpinpografia. Detalle de una fistula vesicocorporal. Visión lateral del paso de contraste entre la cavidad vesical y uterina. (U: Útero; Va: Vagina).

epiplon si fuera necesario. Se realizó una histerectomía total simple por hemorragia profusa secundaria a la lesión de las arterias uterinas durante una de las fistulorrafias. En una paciente se desestimó el rescate quirúrgico dada la expansión local del proceso neoplásico.

Se realizó un seguimiento mínimo de 2 años, manteniéndose las pacientes asintomáticas durante todo este periodo. 2 pacientes lograron mantener la fertilidad y tener otro hijo a partir de los 24 meses de la intervención. La paciente que presentaba el carcinoma de cérvix diseminado falleció a los 14 meses del diagnóstico.

\section{DISCUSIÓN}

La vejiga urinaria está en íntimo contacto con la porción inferior del útero grávido por lo que existe la posibilidad de lesionarla durante la realización de una cesárea segmentaria baja, procedimiento que en las últimas décadas ha ido en aumento. A pesar de estar descritas algunas fístulas vesicouterinas provocadas por agentes etiológicos tan dispares como dispositivos intrauterinos, miomas uterinos necrosados e incluso algún caso producido tras la extracción con vacum ${ }^{8}$, consideramos la yatrogenia provocada por la cirugía obstétrica durante el parto como la causa más importante. Así en la serie de 24 casos descrita por Jòzwik ${ }^{9}$, un $87,5 \%(21 / 24)$ estaban asociados a una cesárea, resultados similares a los publicados por Benchekroun ${ }^{10}$ donde un 76,7\% (23/30) de las fístulas fueron secundarias a una cesárea baja. De nuestras 6 pacientes $5(83,3 \%)$ habían sido sometidas a una cesárea previa.

La presentación clínica de las fístulas vesicouterinas es variable y no está claramente sistematizada. Clásicamente han existido divergencias entre determinados autores en cuanto a qué modelo representaba mejor esta sintomatología, la mayoría basándose en el mecanismo de la continencia urinaria. En un intento de facilitar la compresión de las características clínicas de estas fístulas, Jòzwik en su artículo del 2000 propuso una clasificación basada en el camino que toma el flujo menstrual (Tabla III); el tipo de fístula más frecuente en nuestra serie es la tipo III (50\%) a diferencia de los resultados de este autor donde el 96\% (24/25) son de tipo $\mathrm{I}^{11}$.

\section{TABLA III}

CLASIFICACIÓN CLÍNICA DE JÒZWIK

\begin{tabular}{||l|l||}
\hline Tipo I & - Amenorrea \\
(S. Youssef) & - Menuria \\
& - Continencia urinaria \\
\hline \multirow{2}{*}{ Tipo II } & - Menuria \\
& - Flujo menstrual vaginal \\
& - Incontinencia urinaria \\
\hline \multirow{2}{*}{ Tipo III } & - Flujo menstrual vaginal \\
& - Incontinencia urinaria \\
\hline
\end{tabular}


El diagnóstico en 3 de nuestras pacientes se realiza precozmente al advertir una pérdida de orina a través de la vagina a los pocos días de la cirugía obstétrica, durante el post-operatorio inmediato (tipo III de Jòzwik); esta sintomatología se podría justificar al afectarse el cuello uterino (vesicocervicales) en el $66,6 \%$ de las pacientes (4/6) y al existir un compromiso de la pared vaginal en 2 de estos casos que afectan al cérvix. Sin embargo, en una de las vesicocervicales no fue la incontinencia lo que condujo al diagnóstico, sino el estudio de extensión del proceso neoplásico, que debutó como genitorragia e intensos dolores pélvicos. Según determinados autores ${ }^{2,12}$, las fístulas que comprometen al cuerpo uterino (vesicocorporales) presentan una menor frecuencia de incontinencia por la acción del istmo uterino, que actuaría como un esfínter. A diferencia de las fístulas vesicovaginales, donde la incontinencia es permanente, en las fístulas vesicouterinas la incontinencia urinaria se manifiesta con un curso intermitente. Consecuentemente, en estas pacientes es más prevalente la aparición de menuria (tipo I de Jòzwik). Al no presentar pérdida de orina es mejor tolerada, y por lo tanto, demoraría su diagnostico. En nuestro caso se evidenció a los 3 meses de la intervención. No obstante, otra de las causas que pueden justificar el retraso del diagnóstico en este tipo de fístula vesicouterina es el no poder objetivar la menuria hasta que la amenorrea provocada por la lactancia materna ceda.

Mientras que autores como Jozwik ${ }^{13}$ consideran que alrededor de un 5\% de las fístulas vesicouterinas cicatrizan espontáneamente, en nuestra serie no hemos observado tal resolución en los casos donde se ha aplicado un tratamiento conservador, bien con hormonoterapia bien con cateterización vesical; no obstante, son varios los autores que han publicado la eficacia de estas medidas no invasivas ${ }^{2,3,13-16}$. En ninguno de los casos hemos realizados cauterización de los bordes fistulosos. Al igual que otros autores ${ }^{17,18}$, el tratamiento quirúrgico nos ofrece una mayor garantía de éxito, incluso cuando las pacientes que son diagnosticadas de forma tardía, con márgenes fibróticos, la cirugía ha logrado una alta tasa de buenos resultados ${ }^{19}$.
La presencia de orina en el tracto genital puede disminuir la capacidad reproductiva de la paciente $^{4}$ lo que motiva la consulta médica por esterilidad secundaria, como ocurrió con una de las nuestras 18 años después de una cesárea sin haber presentado previamente ningún otro sintoma urinario. No obstante, en 2 pacientes se produjo un embarazo a término a partir de los 24 meses de la reparación quirúrgica, realizándose una cesárea en el parto sin complicaciones en el seguimiento posterior.

\section{CONCLUSIONES}

La fístula vesicouterina, aún siendo infrecuente, ha dejado de ser un diagnóstico excepcional. Las cesáreas segmentarias bajas constituyen actualmente el mayor factor de riesgo aislado relacionado con el desarrollo de las fístulas vesicouterinas, por lo que debemos tener presente esta lesión en las pacientes que presentan incontinencia urinaria intermitente tras este tipo de cirugía. Recomendamos una reparación quirúrgica diferida sin descartar una actitud conservadora en los casos de pequeño tamaño diagnosticados precozmente.

\section{REFERENCIAS}

1. JOZWIK M, M. JOZWIK, LOTOCKI W.: Actual incidence and cause of vesicouterine fistula. $\mathrm{Br} J$ Urol 1998; 81: 340-343.

2. YIP S, LEUNG T.: Vesicouterine fistula: an updated review. Int Urogynecol J Pelvic Floor Dysfunt 1998; 9 (5): 252-256.

3. TANGER M.: Vesicouterine fistula - a review. Obstet Gynecol Surv 1986; 41 (12): 743-753.

4. LOTOCKI W, JOZWIK M, M. JOZWIK.: Prognosis of fertility after surgical closure of vesicouterine fistula. Eur J Obstet Gynecol Reprod Biol 1996; 64: 8790.

5. YOUSSEF A.: Menuria following lower segment cesarean section. Am J Obst Gynecol 1957; 73: 759-767.

6. LLIXIONA $\mathrm{J}$ et al.: Fistulas vesicogenitales. Rev Esp Obst y Gin 1981; 40: 179-185.

7. SERVER PASTOR G et al.: Tratamiento quirúrgico de las fístulas uretero-vaginales debidas a cirugía ginecológica. Actas Urol Esp 1992; 16: 1-4.

8. YIP S et al.: Vesico-uterine fistula -a rare complication of vacuum extraction in a patient with previous caesarean section. $\mathrm{Br} J$ Urol 1997; 80: 502503.

9. JOZWIK M, M. JOZWIK, LOTOCKI W.: Vesicouterine fistula -an analysis of 24 cases from Poland. Int $J$ Gynecol Obstet 1997; 57: 169-172. 
10. BENCHEKROUN A et al.: Vesico-uterine fistulas. Report of 30 cases. Ann Urol (Paris) 1999; 33 (2): 75-79.

11. JOZWIK M, M. JOZWIK.: Clinical classification of vesicouterine fistula. Int $J$ Gynecol Obstet 2000; 70 (3): 353-357.

12. GÓMEZ CISNEROS S et al.: Fistula vesico-uterina. Arch Esp Urol 1992; 45 (4): 372-373.

13. JOZWIK M, M. JOZWIK.: Spontaneous closure of vesicouterine fistula. Account for effective hormonal treatment. Urol Int 1999; 62 (3): 183-187.

14. RIVAS DEL FRESNO M et al.: Fistula vesico-uterina: a propósito de dos nuevos casos. Actas Urol Esp 1992; 16: 740-742.

15. FERNÁNDEZ RODRÍGUEZ A et al.: Fistula vesicouterina. Tratamiento conservador. Arch Esp Urol 1991; 44 (1): 80-81.
16. ALL-RIFAEI M et al.: Vesicouterine fistula -variable clinical presentation. Scand J Urol Nephrol 1996; 30: 287-289.

17. SOLER SOLER J et al.: Fistula vesico-uterina: aportación de dos nuevos casos. Actas Urol Esp 1998; 22 (2): 146-149.

18. ALLONA ALMAGRO A et al.: Fístulas urinarias: puesta al día. Actas Urol Esp 2002; 26: 776-795.

19. HODONOU R et al.: Vesicouterine fistula: report on 15 cases at Cotonou University Urology Clinic. Prog Urol 2002; 12 (4): 641-645.

Dr. M.A. Bonillo García

C/ Maestro Buenaventura Pascual, 7 - pta. 8 46020 Valencia

(Trabajo recibido el 8 mayo de 2003) 